EPiC Series in Computing
Volume 70, 2020, Pages 79-88
$\begin{gathered}\text { Proceedings of the 12th International Conference } \\ \text { on Bioinformatics and Computational Biology }\end{gathered}$

\title{
Virtual Experimentation Complements Real-World Experimentation
}

\author{
Andrew K Smith ${ }^{1}$, Glen E. P Ropella ${ }^{2}$,and C. Anthony Hunt ${ }^{1}$ \\ ${ }^{1}$ Department of Bioengineering and Therapeutic Sciences, University of California, San \\ Francisco, CA, 94143 \\ ${ }^{2}$ Tempus Dictum, Inc., Milwaukie, OR 97222 \\ drandrewksmith@gmail.com, gepretempusdictum.com, a.hunt@ucsf.edu
}

\begin{abstract}
Studying biological systems is difficult because of complexity, variability, and uncertainty. Conceptual models and diagrams are useful in conveying ideas about how a biological phenomenon are thought to be generated. However, sophisticated modeling and simulation methods are needed to discover mechanism-based explanations. Presented herein is a new and unique methodology for this application. Using virtual experiment methods, we recently provided a plausible solution to a problem that had eluded and perplexed pharmacologists and toxicologists for more than 40 years. We describe how virtual and real-world experimentation can be complementary, and propose a way to partially automate the methodology to expedite research.
\end{abstract}

\section{Introduction}

This paper introduces current and future research methodological ideas, and presents results for a specific application. The first subsection discusses the analogy between biological and software systems. This software system or Biomimetic Software Analog is designed to be biomimetic, and thus analogous to biological systems, e.g. organisms. The second subsection discusses Model Mechanisms (MMs) as hypotheses to explain target phenomenon and their use in the Analogs. The third subsection discusses Virtual Experiments (VEs), which function analogous to real-world experiments. Finally, the fourth subsection discusses the Iterative Refinement Protocol (IRP), the core methodology of Virtual Experiments that tests and selects Model Mechanisms.

The second section discusses a specific application of virtual experiments and the IRP to plasma alanine transaminase (ALT) levels in mice. This research work translates to relevant clinical issues. The final section discusses a proposed Experiment Agent (EA), a semi-autonomous AI that conducts virtual experiments. 


\subsection{Biomimetic Software Analogs}

A biological system, e.g. an organism, is composed of many subsystems organized in space (e.g. organs $\rightarrow$ tissue $\rightarrow$ cells) and time (e.g. transcription before translation), organized hierarchically. A coarse-grained conceptual model of a biological system can be envisioned as a system with a defined interface composed as a hierarchical network of subsystems, each with its own defined interface. Certain objects can move across the interface. This conceptual model is rooted in Thermodynamics, which can be used to further formulate the model. Furthermore, an analogous software system can be designed to have the same structure with information or data crossing the interface. For both biological and software systems, a subsystem can be structural (i.e. a component or part) and/or perform an isolable function. A functional subsystem is composed of interacting parts. In addition, a subsystem is modular, so that it is possible to exchange a subsystem with a different subsystem but the function remains the same.

Both a biological and software systems are networks of subsystems. In our lab, we use a software system to do science, i.e. to increase knowledge by testing and selecting hypotheses. This is done through virtual experimentation, which contains an object of study and its context (see below). In virtual experimentation, it is necessary to compare a software system to the referent biological system of interest. Because the software system is a model or composed of subsystem models of the referent biological system, the strength of the comparison or the strength of the analogy between the two systems gives credibility to the model (i.e. validation). There are two broad categories of validation, which we will characterize as face and structural. Structural validation confirms the model is organized like the referent, and face validation tests whether the model behaves similar to the referent. If it looks like a duck, quacks like a duck, then how can you tell the difference between a virtual or real duck? The virtual duck is a Biomimetic Software Analog (BSA) because it is engineered to look and behave like a real duck, under particular measures.

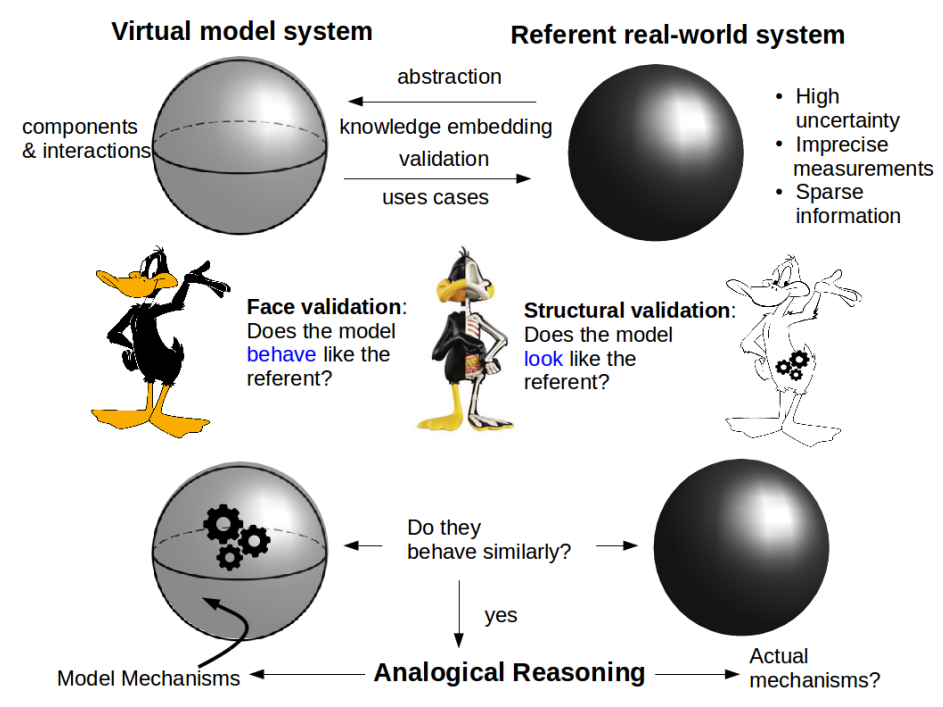

Figure 1. The comparison of a BSA, or virtual model system, to its referent biological system. The BSA is translucent because it is engineered with "gears", the MMs that are thought to generate and explain the phenomena of the referent biological system. We abstract and embed knowledge from the referent biological system into the BSA. Face validation precedes structural validation because of it is more important for the BSA to behave than look like the referent system. We use analogical reasoning to explain the actual mechanisms in the 
real biological system.

The use of models in science is different than the use of models in engineering. Other than testing and selecting hypotheses, models can be used to describe data obtained through wet-lab experimentation or from mining knowledge databases. Bioinformatics is an example of this latter use case. The direct goal is not to explain the data, or develop a mechanism to generate the data, but to describe, analyze, and interpret the data. However, especially with the large bioinformatic datasets, this analysis is a necessary step on the path to explanation.

\subsection{Model Mechanisms for Target Phenomenon}

The details of MMs have been described thoroughly in the literature [1], so the following is a relevant summary. A model is a hypothetical explanation of corresponding targeted phenomenon in the referent biological subsystem. This hypothetical explanatory model is a MM. It is specified by features or parameters, composed of quasi-autonomous components (i.e., software agents), and produces a behavior that can be measured and compared. Also, these measurements and observations of MMs can be compared to wet lab measurements and observations of the referent biological system. There can be a set of equally plausible MMs with each possibly explaining a phenomenon of the referent biological system. The modularity of the software allows these MMs to be variously composed, by substituting alternative modules, and tested through virtual experimentation. A mechanism, virtual or real, is defined [2] as entities and activities organized and orchestrated in such a way that they are responsible for the phenomenon to be explained. A MM emanates from five demanding requirements that guide software engineering, $\mathrm{MM}$ instantiation, and simulation refinements.

1. Five primary characteristics of a biological mechanism - During execution, a MM must exhibit these primary characteristics of a biological mechanism [3]. 1) The MM is responsible for a virtual phenomenon that mimics the biological phenomenon to be explained. 2) It has components (modules, entities, etc.) and activities that are 3) arranged spatially and exhibit structure, localization, orientation, connectivity, and compartmentalization that are (based current knowledge) analogous to biological counterparts. 4) Activities during execution have temporal aspects, such as rate, order, duration, and frequency. 5) The MM has a context, which can include being in a series and/or a hierarchy.

2. Biomimicry - Components and activities are biomimetic facilitate analogical reasoning.

3. Strong parsimony guideline - When scaled, measurements of selected features match or mimic prespecified phenomena to the extent needed to achieve face validation and specific similarity criteria. Adhering to this guideline helps manage the number of equally plausible MMs, while enabling us to increase complexity incrementally. It also facilitates distinguishing a cause from an effect.

4. Emergence - Phenomena measured at a higher level of organization arise mostly from local component interactions and phenomena entanglement at a lower level of organization.

\subsection{Virtual Experiments}

The vision of VEs has been shown in the literature [4]; the following is a relevant summary. VEs is a methodology analogous to wet-lab experiments; a test of an extant MM. The approach is based 
on analogical reasoning [5]. A VE contains the object of study, context, and measurements/observations. The objects of study are the BSAs. The context is any surrounding environment or external influence that may be relevant. Measurements and observations can be obtained to compare to the referent biological experiment. Along with actually performing experiments, virtual experimentation also includes hypothesis formation. In this case, MMs are the hypotheses. A MM supported through virtual experimentation stands as a challengeable yet tested MM-based theory about abstract, plausible mechanism events that may have occurred during the wetlab experiments. To strengthen the virtual-to-wet-lab experiment analogy, virtual features and phenomena are measured analogous to how corresponding wet lab measurements are (or might be) made. Similarly to uncertainty and variability in wet lab experiments, measurements of MMs can exhibit considerable variability as a consequence of Monte-Carlo (MC) sampling and probabilistic specifications, with averages over several MC trials. We envision virtual experimentation as an essential supplement to traditional wet-lab experimentation, an essential part of scientific method. Wet lab experiments provide the data and knowledge for MM formulation and validation, and virtual experiments provide a platform to test hypotheses or explore scenarios unavailable in the wet lab.

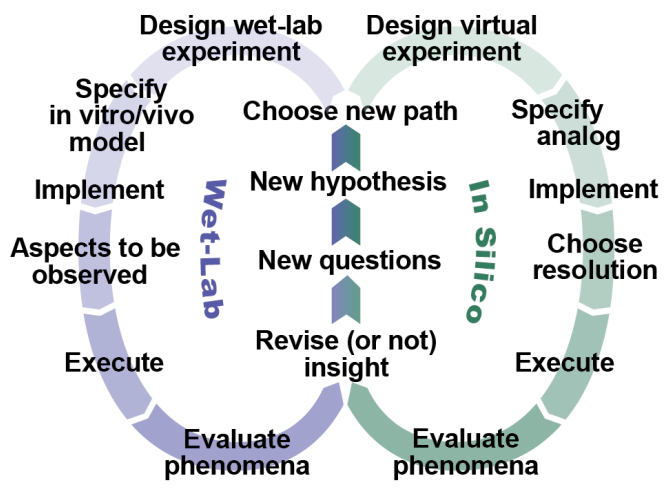

Figure 2: Integrating wet-lab and virtual experiments. The two activities are analogous at every step. When cycling through a particular scientific protocol (wet-lab or virtual), new insights lead to new questions and hypotheses can lead one to switch between wet lab and virtual paths.

\subsection{Iterative Refinement Protocol}

Scientific Method is the cornerstone of science. This methodology involves reasoning, observation, and experimentation. Scientific Method can be described in the following steps (assuming a problem of interest or attribute in a natural system targeted for understanding):

1. Use reasoning (e.g. inductive, analogical, abductive) and current knowledge to develop a set of plausible mechanistic hypotheses.

2. Test the predicted behavior of a hypothesis through experimentation using observations and measurements. Evidence is developed through deductive reasoning and aggregated using induction.

3. The hypothesis is either rejected because it is falsified, or accepted:

a. Acceptance here is that the hypothesis has been supported, not that it is true.

b. If rejected, then choose another hypothesis and repeat from first step. 
Falsification is important because it is the only way to eliminate the space of hypotheses (i.e. a member of the set). A possible way of using abductive reasoning to rank the remaining accepted hypotheses thereby furthering the selection is by Bayesian Inference (see Subsection 3.4).

The Iterative Refinement Protocol (IRP) simulates scientific method for falsifying, refining, and validating explanatory MMs. Because of multisource uncertainties, to achieve validation it is essential to conduct many narrowly focused virtual experiments to incrementally and systematically shrink the constellation of plausible MM parameterizations. The IRP consists of the following steps:

1. Assemble \& prioritize a diverse set of wet lab observations and measurements to be explained or simulated called Targeted Attributes (TAs), then update use cases. Choose a TA for the current cycle. This choice is constrained on the available data and knowledge, and the particular use case. Formulate a MM (or a set of a variety of MMs), determine its specification (measurements \& granularity). Specify the process of comparison to the referent and the requisite degree of similarity that must be achieved (Similarity Criteria (SC) \& TA measurements), which can range from qualitative (e.g. event $\mathrm{X}$ occurs before event $\mathrm{Y}$; temporal profiles have a sigmoidal shape) to quantitative (e.g. simulated measurements are within $10 \%$ of wet lab measurements).

2. Implement and verify MM (either vary parameters or revise existing MM components and modules) using parsimony, set up and run the simulations, record measurements.

3. Selection and iteration:

a. If MM is falsified (new knowledge), then return to step 2.

b. If MM accepted (achieve TAs \& SC and thus a degree of validation), then return to step 1 and either increase SC stringency choose another TA.

The method is iterative in two ways, one over the set of plausible MMs for one TA, and one over a set of TAs. The iterative process of falsification-refinement-validation ensures that the MM is increasingly biomimetic yet parsimonious. MM credibility increases by increasing the strength and variety of validation targets. At corresponding degrees of granularity, the MM during execution and the actual biological mechanism may be strongly analogous within and across multiple levels. That analogy is only as strong as the weakest link in the networked MM during execution, and that depends first on use case-dependent virtual-to-actual mapping similarities. MMs are perpetual works in progress, not finished products. We envision MMs being improved incrementally through multiple future rounds of MM challenge and validation against an expanding set of TAs.

\section{Example: Virtual Experiments on ALT release Model Mechanisms}

This section describes a recent application of virtual experimentation to the understanding of drug induced liver injury, and in particular the chemical biomarker ALT. The model biological system is the release of ALT into the bloodstream following a toxic dose of the drug acetaminophen (APAP). The first subsection describes a virtual mouse as the biomimetic software analog. The second subsection describes the Model Mechanism implemented to explain the plasma ALT levels measured in real mice (the Targeted Attribute). The third subsection discusses the results from virtual experimentation. The fourth subsection discusses future work in this research project. 


\subsection{Virtual Mouse}

A virtual mouse or Mouse Analog is a concrete, biomimetic software system engineered to look and behave like an real mouse (Fig. 1A-D). It comprises Mouse Body, Liver, and a space to contain Dose for simulating intraperitoneal dosing. Liver is the number of Monte Carlo-sampled Lobule variants per experiment and is strongly analogous to actual livers across several anatomical, lobular, and cell biological characteristics. A Lobule comprises a directed acyclic graph with a particular SS object at each graph node. Flow follows the directed graph. Graph nodes are organized into three Layers, which map to conventional hepatic zones; with the number of SSs decreasing from Layer 1 to 3. That structure maps directly to the quasi-polyhedral nature of hepatic lobules. All SS dimensions are Monte-Carlo sampled within constraints. Each SS comprised of multi-layered, cylindrical spaces. Events, such as adsorption, diffusion, convection, and metabolism, occurring within a particular SS are analogous to microscopic referent events occurring within portions of sinusoids and adjacent tissue. Entry and exit of chemical objects from Endothelial Cells and vHPCs is mediated by the Cell (which is a software agent). The virtual mouse has achieved qualitative and quantitative validation targets through previous IRP cycles.
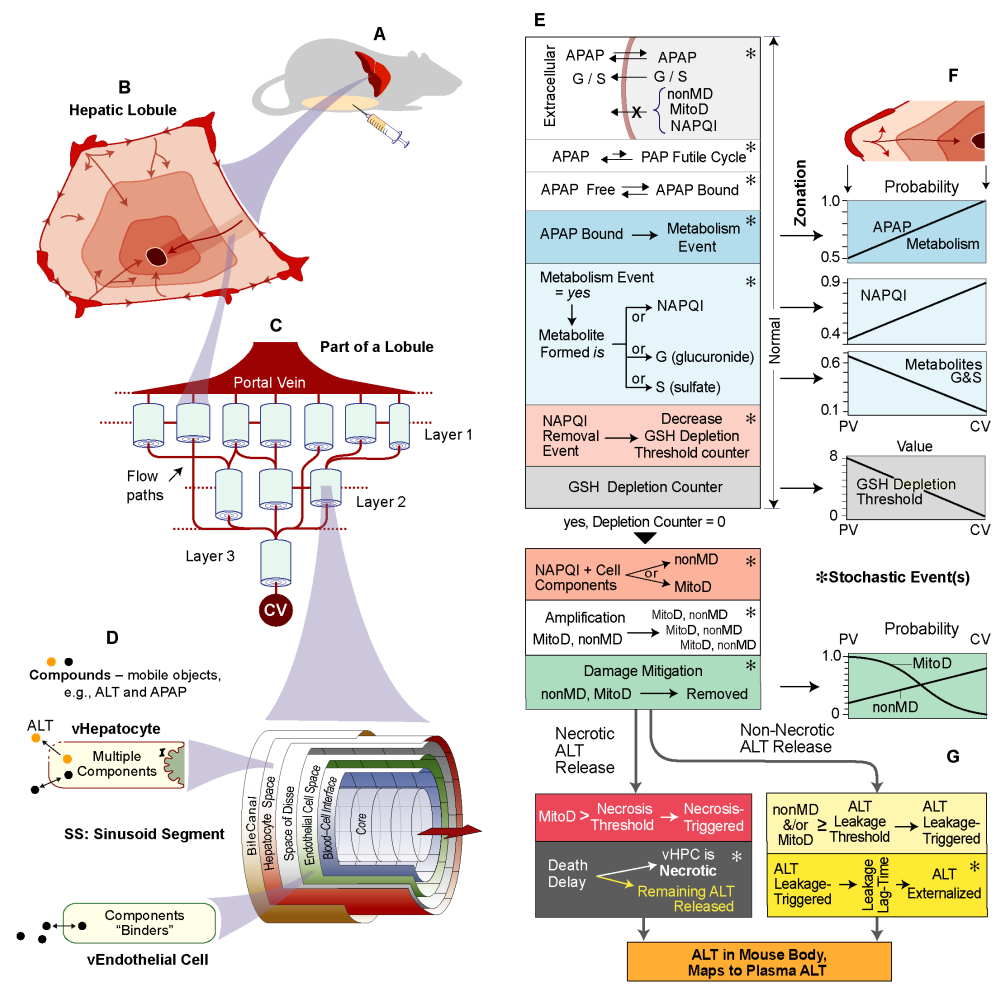

Figure 3: Component organization and mechanism features. (A) A virtual Mouse is a concretized, coarsegrain software analogy of a real mouse . (B) Shading illustrates hepatic zonation. (C) A Monte-Carlo specified interconnected directed graph specifies flow paths for chemical objects. (D) A multi-layered Sinusoid Segment (SS) maps to a portion of hepatic tissue. One is placed at each graph node. A space within an SS contain Virtual Hepatocytes (vHPCs), which contain a variety of objects enabling cause-effect events within the Mechanism . (E) Each of these events and activities (along with those in $\mathbf{F}$ and $\mathbf{G}$ ) may occur each simulation cycle. Events in the top series, labeled "Normal," occur following therapeutic (non-toxic) and toxic doses of APAP. Those listed 
subsequently contribute to simulated APAP-induced injury. (F) Many events have location-dependent probabilistic parameterizations. There is a direct mapping between the probability of an APAP Metabolism event and average metabolic capacity at various lobular locations. Each vHPC uses values drawn from those gradients. (G) The logic of the ALT release MM is shown (see text).

\subsection{Model Mechanism of ALT release from Hepatocytes}

For all experiments we utilize a previously parameterized, validated spatiotemporal MM, the parent MM (Fig. 3 E,F), that explains the characteristic pattern of necrosis following a toxic APAP dose in mice [6]. The new TA for this work is plasma ALT levels in mice. Therefore, we want to add and implement hypothetical MMs for ALT release, guided by current information from the literature, to the parent MM. Gamal et al. [7] described leakage of cytoplasmic material associated with APAPinduced disruption of hepatocyte integrity and surface damage associated with blebbing. Therefore, the working hypothesis is ALT is released by two separate processes, passive release through necrosis and non-necrotic (i.e. damage) release. Because there is no strong wet lab evidence to the contrary, we assumed that each hepatocyte, independent of lobular location, contains the same amount of ALT. The parent MM in Fig. 3E includes a Necrotic state for a vHPC; therefore, instantiating a virtual counterpart to ALT release caused by necrosis was straightforward. When a vHPC transitions to Necrotic, all remaining ALT is released and externalized (bottom, Fig. 3G). Externalized ALT objects follow the same stochastic movement rules as other chemical objects, including entering the Body. An ALT-in-Mouse-Body is scaled directly to represent plasma ALT. We name that entire sequence, from Metabolism to ALT externalization, the Necrosis-Model. For simplicity, ALT-in-Mouse-Body is not removed, and accumulates in the Body. We sought parsimonious extensions of the parent MM that 1) could map to the non-necrotic ALT release process, 2) would operate concurrently with-but independent of - Necrosis-Model, and 3) make ALT externalization a direct function of Damage Products. The parent MM in Fig. 3E includes generation of two types of Damage Products, nonMD (maps to all types of non-mitochondrial damage products) and MitoD (maps to all types of mitochondria related damage products) within each vHPC. We instantiated three versions of the coarse grain Non-Necrotic ALT Release process illustrated in Fig. 3G by making ALT externalization a direct function of the amount of Damage Products in each vHPC: nonMD, MitoD, or both. When MitoD (nonMD) is the cause of ALT Externalization, we name the MM Mito-D-Model (nonMDModel). When both cause of ALT Externalization, we name the MM Dual-Model. Each simulation cycle, each vHPC determines if its amount of designated Damage Products exceeds an ALT Leakage Threshold value. If so, that vHPC becomes Leakage-Triggered and initiates an ALT externalization process. The vHPC specifies a Leakage Lag-Time by a pseudo-random draw from a uniform [Min, Max) distribution. When the Lag-Time duration is reached and the vHPC's ALT counter value $>0$, the vHPC creates an ALT object, externalizes it, and decrements its ALT counter by 1 . For NecrosisModel, when a Necrosis-Triggered vHPC transitions to Necrotic, and its ALT counter value $>0$, the vHPC creates ALT objects corresponding to the counter value and externalizes them with zero-time delay.

\subsection{Results from Virtual Experimentation}

The objective of this work is to posit plausible cause-effect linkages between APAP disposition and metabolism and concurrent measurements of ALT in plasma. Until sublobular hepatocyte damage and ALT release can be measured concurrently at multiple times within the same subject, it will be infeasible to establish those linkages in vivo. The alternative approach developed herein is to use virtual experiments to challenge and falsify (or not) many potentially explanatory, biomimetic concretized MM-based hypotheses [1]. Early workflow results suggested that ALT externalization and accumulation in Mouse Body as a consequence of Necrosis-Model executions could not scale quantitatively to closely match the temporal patterns of plasma ALT, the validation targets. Results 
from experiments to achieve stringent validation targets used MitoD-Model because we judged it to be the more parsimonious of the three. We also observed no explanatory advantage when using nonMD-Model or Dual-Model.

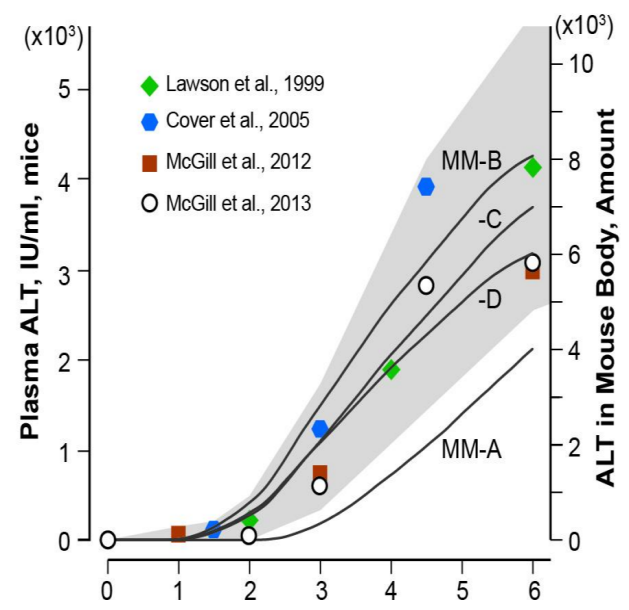

Figure 4: The gray area is the validation target range (left axis), which is based on mouse data from the four indicated reports. The SC for an acceptable ALT release MM is that it generates ALT-in-Mouse-Body profiles that, when scaled (right axis), fall within the target range. The four labeled profiles were generated by the MMs described in the text.

\subsection{Falsification using another Targeted Attribute}

The MM with ALT release from both necrotic and non-necrotic hepatocytes caused by mitochondrial damage satisfied the similarity criteria for plasma ALT levels measured from real mice. In following the IRP, the next step to validate or falsify this MM by testing against another TA. One such measurement is protein adducts caused by APAP metabolism. If a normal process is damaged during APAP-induced injury, its selectivity may be eroded leading to concomitant externalization of other macromolecules, including ALT. Consistent with that scenario, McGill et al. [8] reported that, following a low toxic APAP dose (no necrosis), APAP-protein adducts are externalized to blood prior to ALT elevations. These adducts map to the damage products produced by hepatotoxicity mechanisms in the virtual mouse. Measurements of these adducts over time can be used as TA for further cycles of the IRP.

\section{Experiment Agent or Automated Experimentation}

This section discusses future advances that we believe will help strengthen synergies between wetlab and virtual experimentation, thus expediting research efforts. Performing many cycles of the IRP can be tedious and time consuming; automation would help. Therefore, we propose to design a software agent to perform the experiments, or an artificially intelligent "scientist." The first subsection discusses why humans are still needed for hypothesis formulation. The second subsection discusses possibly using machine learning to search the space of MMs and the automation of the IRP. The third subsection discusses ways to create SCs. Finally, the fourth subsection discusses using Bayesian Inference to select and rank MMs. 


\subsection{Humans for hypothesis formulation}

Hypothesis formulation is largely an abductive reasoning process. Because of this, humans are still better than AI. However, along with expert opinion, AI could be used to mine databases so that current knowledge and data used to form hypotheses could more easily be obtained and analyzed [9]. In our lab, we are considering setting up a database of possible TAs and virtual experimentation results. Along with hypothesis formulation, this database could be used to gather data and develop TAs for VE. This is also a time-intensive, manual process because there are many wet lab experiments with slightly different contexts and considerable uncertainty among a set of similar experiments.

\subsection{Automation of the IRP and AI for searching}

Once a process can be written down as a series of steps then either full or partial automation can be conceived. The IRP from above can be simplified by the following steps:

1. Hypothesis formulation, as a MM, and implementation (also verification)

2. Vary or search MM, and test against TA with SC.

3. Reject or accept and rank MMs

As mentioned above, step 1 can be partially automated. However, step 2 can potentially be fully automated similar to model fitting. We currently have a random search method in which a range of values or set of specifications are inputted for relevant parameters and a random selection is made. For large parameter spaces this becomes unwieldy quickly; therefore, information from the referent or parameters would be useful to shrink the space of the search. There is current work in the lab to search parameter space for regions where the output behavior (i.e. the one compared to the TA) changes quickly or nonlinearly, and for regions of relatively little change (i.e. robustness). This exploration, similar to a sensitivity analysis, can be automated.

\subsection{Jensen-Shannon Divergence as a Similarity Criterion for Testing}

The estimation of similarity between any 2 traces/outputs from a MM can be broadly divided into 2 types of approach: theoretically justified versus ad hoc. In [10] an attempt was made to justify the calculation of similarity by various distance measures. To fully justify such methods theoretically would require the formulation of $\mathrm{MM}$ behavior as a metrizable space, which can be prohibitively expensive for exploratory models like ours. Parametric statistical comparisons also require some justification. For example, comparing means and standard deviations assumes Gaussian distributions, which is not guaranteed by our MM, which composes pseudo-randomly sampled distributions, most of which are uniform, but some of which are not. The properties of the Jensen-Shannon Divergence (JSD) can be used agnostically to compare any 2 time series, when normalized and interpreted as a probability density function, to produce an aggregate value. Along with other estimates of similarity, JSD can provide a very coarse ad hoc, but fast and automatic, SC. See [11] for an overview.

\subsection{Model Selection by Bayesian Inference}

In complex situations, selecting a member from a set of accepted MMs is not easy because of uncertainty. Model selection using Bayesian Inference has been applied to statistical models [12], but not to MMs. A MM has a certain probability for being plausible a priori based on current knowledge, logic, etc. After VE, if the MM is validated, then that probability increases. Most manually varied parameters fail to satisfy the SC, but some do, and within this set some do "better" than others. Let M $=$ one $\mathrm{MM}$ out of the set $\{\mathrm{MM}\}, \mathrm{Y}=$ validated $\mathrm{MM}$ output from $\{\mathrm{Y}\}, \mathrm{D}=\mathrm{TA}$. The probabilities are the following: 1) $\mathrm{P}(\mathrm{M} \mid \mathrm{D})$, probability of the $\mathrm{MM}$ given the TA, 2) $\mathrm{P}(\mathrm{M} \mid \mathrm{Y})$, probability of the $\mathrm{MM}$ 
given the MM output, 3) $\mathrm{P}(\mathrm{D})$, probability of the TA, 4) $\mathrm{P}(\mathrm{Y} \mid \mathrm{M})$, probability of the MM output given the $\mathrm{MM}$, 5) $\mathrm{P}(\mathrm{Y})$, probability of the $\mathrm{MM}$ output, and 6) $\mathrm{P}(\mathrm{MM})$, probability of the MM. The goal is to update or infer $\mathrm{P}(\mathrm{M} \mid \mathrm{D})$ with information from matching $\mathrm{Y}$ to the TA using the SC every IRP cycle using Bayes' Rule, $\mathrm{P}(\mathrm{M} \mid \mathrm{Y}) * \mathrm{P}(\mathrm{Y})=\mathrm{P}(\mathrm{Y} \mid \mathrm{M}) * \mathrm{P}(\mathrm{M})$, giving a certain likelihood that can be used to rank the set of models. In addition, we would like to have a probability suggesting the likelihood that a particular $\mathrm{MM}$ is adequately explanatory. As more information from successive experiments accumulates, we can update this probability.

\section{References}

[1] Hunt CA, Erdemir A, Mac Gabhann F, Lytton WW, Sander EA, Transtrum MK, et al. The spectrum of mechanism-oriented models for explanations of biological phenomena. Processes. 2018;6(5):56

[2] Illari PM, Williamson J. What is a mechanism? Thinking about mechanisms across the sciences. Eur J Philos. Sci. 2012;2:119-35

[3] Darden L. Thinking again about biological mechanisms. Philos Sci. 2008;75:958-69

[4] Petersen BK, Hunt CA. Developing a vision for executing scientifically useful virtual biomedical experiments. In: Proceedings of the 2016 Spring Simulation Multiconference; 2016; p. 697-706, Society for Computer Simulation International, San Diego

[5] Bartha P. "Analogy and Analogical Reasoning", The Stanford Encyclopedia of Philosophy (Spring 2019 Edition), Edward N. Zalta (ed.), URL https://plato.stanford.edu/archives/spr2019/entries/reasoning-analogy/

[6] Smith AK, Petersen BK, Ropella GE, Kennedy RC, Kaplowitz N, Ookhtens M, et al. Competing mechanistic hypotheses of acetaminophen-induced hepatotoxicity challenged by virtual experiments. PLoS Comp Biol. 2016;12:e1005253

[7] Gamal W, Treskes P, Samuel K, Sullivan GJ, Siller R, Srsen V, Morgan K, Bryans A, Kozlowska A, Koulovasilopoulos A, Underwood I. Low-dose acetaminophen induces early disruption of cell-cell tight junctions in human hepatic cells and mouse liver. Scientific reports. 2017 Jan 30;7:37541

[8] McGill MR, Lebofsky M, Norris HR, Slawson MH, Bajt ML, Xie Y, et al. Plasma and liver acetaminophen-protein adduct levels in mice after acetaminophen treatment: dose-response, mechanisms, and clinical implications. Toxicol Appl Pharmacol. 2013;269:240-9

[9] Spangler S, Wilkins AD, Bachman BJ, Nagarajan M, Dayaram T, Haas P, Regenbogen S, Pickering CR, Comer A, Myers JN, Stanoi I, Kato L, Lelescu A, Labrie JJ, Parikh Neha, Lisewski AM, Donehower L, Chen Y, and Lichtarge O. 2014. Automated hypothesis generation based on mining scientific literature. In Proceedings of the 20th ACM SIGKDD international conference on Knowledge discovery and data mining (KDD '14). ACM, New York, NY, USA

[10] Ropella G.E.P., Nag D.A., and Hunt C.A.. Similarity measures for automated comparison of in silico and invitro experimental results. In Proceedings of the 2003 IEEE Engineering in Medicine and Biology Society Conference, Cancun, Mexico, September 2003. IEEE

[11] Cha, Sung-Hyuk. 2007. "Comprehensive Survey on Distance/Similarity Measures Between Probability Density Functions.” International Journal of Mathematical Models and Methods in Applied Science 1 (4):300-307

[12] Murakami Y. Bayesian Parameter Inference and Model Selection by Population Annealing in Systems Biology. PLoS One; 9(8): e104057 